\title{
Schlüsselfaktoren im Marketingkonzept von Schweizer Gig-Workern
}

\author{
Tobias Burri und Etienne J. Rumo
}

\section{Zusammenfassung}

Gig-Worker sind Arbeitskräfte, die ihre Dienstleistungen auf Internetplattformen anbieten und dadurch für verschiedene Aufraggeber tätig sein können. Jeder Auftrag wird als „Gig“ bezeichnet. Diese wachsende Form der Beschäftigungsart hat dazu geführt, dass Gig-Worker ähnlich einem Einzelunternehmen selbstständig agieren. Dementsprechend ist auch unternehmerisches Denken und Handeln erforderlich. Marketingaktivitäten, wie z. B. die Erstellung und Ausführung eines Marketingkonzeptes, sind ein wichtiger Bestandteil davon. Dieses Kapitel soll Gig-Workern nützliches Grundwissen anbieten und gleichzeitig eine Vorlage für zukünftige Marketingkonzepte sein. $\mathrm{Zu}$ Beginn werden das Thema „Gig-Work“, dessen wachsende Verbreitung und die Problemstellung dieser Arbeit vorgestellt. Später werden klassische und aktuelle Theorien zum Marketing und dessen Konzepte erklärt und anschließend der Bezug zu Gig-Workern in der Schweiz hergestellt.

T. Burri $(\bowtie)$

Bern, Schweiz

E-Mail: tobias@innoarchitects.ch

E. J. Rumo

Berner Fachhochschule Wirtschaft, Bern, Schweiz

E-Mail: etienne.rumo@bfh.ch 


\subsection{Einleitung}

Die Digitalisierung und die damit verbundene sogenannte Arbeit 4.0 verursachen massive Veränderungen der Geschäftsmodelle und somit auch in der Arbeitswelt. Das Internet bildet vermehrt das Zentrum der strategischen Ausrichtung von Unternehmen (Stähler 2002, S. 32) und auch von Menschen, die über Plattformen im Internet selbstständig nach Aufträgen suchen und diese gegen Bezahlung erledigen. „Gig-Worker“ ist ein Anglizismus der sich aus den Worten Gig (Soloauftritt) und Worker (Arbeitnehmer) ableitet. ${ }^{1}$ Der Arbeitnehmer erledigt seine Aufträge (Auftritte) alleine für verschiedene Auftraggeber, welche ihn über eine Plattforme beauftragen.

In einer der aktuellsten Übersichten von Gig-Workern in den USA ist zu entnehmen, dass $16 \%$ der Amerikaner bereits mindestens einmal in ihrem Leben einen Dienst über das Internet angeboten haben (Shahdad und Seidenstein 2017).

Im Kontext der Gig Economy nimmt die Sharing Economy (englisch für Wirtschaft des Teilens) einen wichtigen Platz ein, diese ist jedoch nicht Gegenstand dieser Untersuchung. Der Fokus liegt auf Menschen, die ihre Arbeitskraft auf Plattformen anbieten und nicht auf Menschen, die ihren Besitz über Plattformen mit anderen gegen Bezahlung teilen.

Auffallend in dieser Statistik sind der hohe Ausbildungsgrad sowie die Altersverteilung von Gig-Workern in Amerika. Gut ausgebildete junge Menschen versuchen vermehrt in der Gig Economy Arbeit zu finden, um so ihren Lebensunterhalt zu bestreiten (Risak und Lutz 2017, Kap. 3).

Die Schweiz zählt gemäß einer von Upwork in Auftrag gegebenen Studie zu den wachstumsstärksten Ländern im Bereich der Anstellung von Gig-Workern (Upwork 2015). Junge und oftmals gut ausgebildete Menschen sowie jene, die sich noch in der Ausbildung befindend versuchen mittels Gig-Work nebst der finanziellen Motivation, auch ihre berufliche Zukunft zu gestalten. Durch die Arbeit als Gig-Worker können sie Arbeitserfahrung, ein berufliches Netzwerk und bereits einen Kundenstamm aufbauen (McGovern 2017).

Im Vergleich zu Amerika ist der Anteil in der Schweiz von Erwerbstätigen in sogenannt atypisch-prekären Arbeitsverhältnissen seit 2004 stabil zwischen $2 \%$ und $3 \%$. Gemäß Mattmann et al. (2017) ist es eine Tatsache, dass ein Großteil der Gig-Worker mit ihrer Arbeit weniger als den Schweizer Medianlohn verdienen. Auch im Ausbildungsgrad der Gig-Worker unterscheidet sich die Schweiz von Amerika. Die vom Staatssekretariat für Wirtschaft in Auftrag gegebene Studie besagt, dass vorwiegend Frauen, jüngere Erwerbstätige sowie Männer mit tiefem Bildungsniveau in der Gig Economy tätig sind.

\footnotetext{
${ }^{1}$ Durch die Übernahme des englischen Ausdrucks ist auch die geschlechtsneutrale Formulierung gegeben. In dieser Arbeit steht Gig-Worker stets für weibliche und männliche Arbeitnehmerinnen und Arbeitnehmer. Weitere geschlechtsbezogene Nennungen werden aus Gründen der leichteren Lesbarkeit in der gewohnten männlichen Sprachform verwendet. Dies impliziert jedoch keine Benachteiligung des weiblichen Geschlechts, sondern soll im Sinne der sprachlichen Vereinfachung als geschlechtsneutral zu verstehen sein.
} 
Die Schweiz zählt zu den 10 Ländern weltweit, die im Verhältnis zur Größe der Volkswirtschaft am meisten Gig-Worker haben. Es ist anzunehmen, dass wie auch in Amerika, diese Zahl in Zukunft noch ansteigen wird. Um als Gig-Worker erfolgreich zu sein, muss die Person sich nebst den administrativen Aufgaben auch sehr spezifischen Herausforderungen, wie z. B. dem digitalen Marketing und Branding (englisch für Markenbildung), stellen.

\subsection{Problemstellung}

Aus der Arbeit von Burri, Figni und Rosa (2018) geht hervor, dass Gig-Worker wie selbstständig Erwerbende und Unternehmen über unternehmerisches Denken und Handeln verfügen müssen. Gig-Worker sind sich dessen im Unterschied zu den anderen selbstständig Erwerbenden und Unternehmern jedoch nicht zwingend bewusst. Ein Gig-Worker wird in dieser Arbeit als eine Person definiert, die ihre Aufträge über eine oder mehrere Plattformen erhält, welche für sie auch den gesamten Geldfluss übernehmen. Die Arbeit als Gig-Worker hat gegenüber der Arbeit in einem Anstellungsverhältnis den Vorteil, dass die Arbeitszeit und die Menge an Arbeit durch den Gig-Worker selbst eingeteilt und bestimmt werden kann. Hingegen erhält der Gig-Worker kein fixes Einkommen. Die Stabilität eines geregelten Arbeitsverhältnisses und Zusatzleistungen wie ein Bonus oder ein 13. Monatsgehalt sind in der Gig Economy nicht gegeben.

Gemäß einer von der Unternehmensberatung Deloitte durchgeführten Studie mit 4000 Gig-Workern in den USA versuchen viele Gig-Worker nach einiger Zeit wieder als Arbeitnehmer bei einem Unternehmen Fuß zu fassen. Grund dafür ist die fehlende Unternehmenskultur im Gig-Anstellungsverhältnis (Deloitte 2016). Damit ein Gig-Worker wirklich erfolgreich sein kann, muss er, zusätzlich zu seiner Haupttätigkeit als Fachkraft, generelle unternehmerische Aufgaben wie Strategieerstellung, Finanzierung, Marktanalysen, Administration etc. wahrnehmen (Merath 2008, S. 220 ff.). Klassische Marketingtätigkeiten sind hier ebenfalls zu nennen. Ist der Gig-Worker nicht bereit oder fähig, diese Marketingaktivitäten selber wahrzunehmen, ist er auf externes Wissen angewiesen. Die Komplexität dieser Aufgaben und der damit verbundene Zeitaufwand bilden die Hauptprobleme, weshalb Gig-Worker ihre Arbeit oftmals wieder verlassen um in ein festes Anstellungsverhältnis zurückzufinden. Die Problemstellung dieser Arbeit ist auf den Bereich Marketing fokussiert. Die Arbeit hat zum Ziel, das notwendige Wissen für Gig-Worker bereitzustellen, damit sie ihr Marketing selbstständig übernehmen können.

\subsection{Gig-Work-Plattformen}

Eine Gig-Work-Plattform bildet das Bindeglied zwischen dem Gig-Worker und dessen Auftraggebern. Als Unternehmen stellt es Online-Dienstleistungen zur Verfügung, die sowohl von Gig-Workern als auch deren Kunden bzw. Auftraggeber genutzt werden können. 
Gig-Work-Plattformen unterscheiden sich einerseits durch ihre angebotenen Dienstleistungen und andererseits durch ihre Ausrichtung. Als bekanntes Beispiel kann man die Firma Uber nennen. Uber ist eine Plattform, auf der sich Gig-Worker als Fahrer registrieren können. Diese Gig-Worker werden durch die Plattform von Uber mit Kundenaufträgen zum Personentransport versorgt. Der Fahrgast bezahlt via App (Mobile Applikation) die Plattform für den Fahrdienst. Diese leitet dann wiederum den Betrag abzüglich einer eigenen Provision an den Fahrer weiter. Durch dieses Geschäftsmodell ist Uber zum weltgrößten Taxibetrieb geworden, ohne dabei weder die Autos zu besitzen noch die Fahrer anzustellen (Jaekel 2017, S. 161 ff.). Nebst dieser auf Taxifahrten ausgerichteten Plattform existieren auch Plattformen, die Gig-Worker aus verschiedenen Berufs- und Tätigkeitsfeldern vermitteln. Die Plattform Upwork hat sich auf Talente in den Bereichen der Administration, Buchhaltung, Marketing, Design und Web-Development spezialisiert (Upwork Global 2018).

\subsubsection{Geschäftsmodelle der Plattformen}

Das klassische Gig-Economy-Geschäftsmodell zeichnet sich dadurch aus, dass der Kunde, der eine Dienstleistung nutzt, jeweils direkt die Plattform dafür bezahlt. Diese leitet den Betrag abzüglich einer Provision an den Gig-Worker weiter. Der Gig-Worker selbst muss Vorgaben zur Teilnahmeberechtigung bei der Plattform einhalten und teilweise auch finanzielle Leistungen für die Teilnahme entrichten. Dieses Geschäftsmodell sieht sich in der Schweiz vermehrt mit Vorwürfen konfrontiert, die soziale Verantwortung eines Arbeitgebers nicht richtig wahrzunehmen. Die Plattformen hingegen erachten ihre Gig-Worker als selbstständig Erwerbende und nicht als Angestellte, da diese keinen Anstellungsvertrag bei ihnen unterzeichnet haben (Schöchli 2016).

Verdeutlicht wird dies z. B. am Geschäftsmodell von Uber (Osterwalder und Pigneur 2011, S. 169; Oakley 2016). Im Bereich Key Activities (englisch für Schlüsselaktivitäten) kann man dort entnehmen, dass die Plattformentwicklung, das Marketing und die Gig-Worker-Verpflichtung priorisiert behandelt werden. Die Plattform mietet das Auto vom Fahrer und bezahlt diesen für die geleisteten Fahrten. Eine 2. Ausrichtung des Geschäftsmodelles verläuft vom Zahlungsfluss her ähnlich, unterscheidet sich jedoch von der 1. hinsichtlich den Zusatzdienstleistungen, die sie für ihre Gig-Worker übernimmt. Wie üblich bezahlt auch hier der Auftraggeber direkt bei der Plattform für die geleistete Arbeit oder Dienstleistung des Gig-Workers und die Plattform leitet anschließend ein Honorar an den Gig-Worker weiter. Anstelle einer Provisionsbasis für die Plattform bezahlt der Gig-Worker einen fixen Preis für gewisse Dienstleistungen die er von ihr in Anspruch nimmt. So führt die Plattform für ihn die Lohnbuchhaltung und übernimmt damit auch Zahlungen an die Sozialversicherungen, Krankentaggelder und die berufliche Vorsorge. Der Gig-Worker ist auch in diesem Geschäftsmodell nicht ein Angestellter der Plattform, sondern bezahlt mit einem Teil seines Lohnes vom Auftraggeber die Plattform für die Übernahme dieser Dienstleistungen (Burri et al. 2018). Ein Gig-Worker muss daher bei 
der Wahl der geeigneten Plattform darauf achten, welche Konditionen und Leistungen für ihn gelten und selbst bestimmen, bei welchen Plattformen seine Dienstleistungen angeboten werden sollen. Die 2. Ausrichtung kann dem Gig-Worker administrative Arbeiten und unternehmerische Tätigkeiten abnehmen. Zudem sind dabei die soziale Absicherung und Vorsorge gewährleistet. Dies bietet Vorteile bei längerer Zeit ohne Aufträge, einem Unfall oder Krankheit. Es hat aber auch den Nachteil, dass abhängig von den Gesamtkonditionen weniger Gewinn pro erledigten Auftrag verdient wird.

Diese Ausrichtung von Plattformen bringt den Gig-Worker sehr nahe zum Typus des Freelancers (englisch für Freiberufler) oder der temporären Arbeitskraft, die wie üblich über eine Agentur an ihre Arbeitgeber vermittelt wird (Bürgi, Nägeli Rechtsanwälte 2008). Einer der Hauptunterschiede dieser Plattformen zu Vermittlungsagenturen ist aber, dass üblicherweise die Vermittlungsagenturen die Entscheidung treffen, welche Arbeitskraft sie dem Auftraggeber zur Verfügung stellen. Bei der Plattform wählt der Auftraggeber direkt den Gig-Worker aus, um ihn mit einer Tätigkeit zu beauftragen.

Tab. 14.1 zeigt eine Übersicht der existierenden und aufstrebenden Geschäftsmodelle von Plattformen in der Sharing Economy. Die Gig Economy ist ein wesentlicher Bestandteil der Sharing Economy, in welcher die Plattformen die zentrale Rolle der Verbindung zwischen den Nutzern, der Kunden, den Suchenden und den Auftraggebern herstellen. Die beiden beschriebenen Geschäftsmodelle sind in der untenstehenden Tabelle gegenwärtig meist im violetten und zukünftig auch im roten Bereich einzuordnen.

Die Plattformen übernehmen die Funktion der Ressourcenallokation der verfügbaren Arbeitskraft auf die Nachfrage an Arbeitsleistung und überwachen dabei den Gesamtprozess. Es erlaubt vor allem Gig-Workern, Aufträge zu akquirieren und erleichtert den Auftraggebern den Such- und Anstellungsprozess.

In Bezug auf das Marketingkonzept von Gig-Workern bedeuten diese verschiedenen Formen von Geschäftsmodellen nun, dass der Mehrwert eines Gig-Workers verschieden wahrgenommen werden kann. Je nach Geschäftsmodell kann der Kunde den Gig-Worker z. B. anhand seiner bisherigen Bewertungen oder der schnellst möglichen Verfügbarkeit direkt buchen, der Gig-Worker wiederum hat die Möglichkeit, sich für die auf der Plattform ausgeschriebenen Gigs mittels einer Offerte zu bewerben. Der Gig-Worker in einem solchen Geschäftsmodell ist also darauf bedacht, sein Profil auf der Plattform so zu gestalten, dass er für Kunden attraktiv wirkt. Dabei müssen seine Bewertungen so hoch wie möglich sein.

\subsubsection{Marketing der Plattformen}

Obwohl nicht jede Plattform dieselben Marketingmaßnahmen betreibt, so stützen sie sich jedoch alle auf ähnliche, für Gig-Worker relevante, Marketinginstrumente und -aktivitäten.

Basierend auf der Entscheidung, nach welchem Geschäftsmodell eine Plattform ausgerichtet wird, ergeben sich unterschiedliche Mehrwerte für die involvierten Anspruchsgruppen. Ist das Plattformziel, wie z. B. bei Uber, für eine ganz bestimmte Dienstleistung 
Tab. 14.1 Geschäftsmodelle von Plattformen

\begin{tabular}{|c|c|c|c|}
\hline Traditional & Platform & Sharing Platform & $\begin{array}{l}\text { Co-Operative Sharing } \\
\text { Platform }\end{array}$ \\
\hline $\begin{array}{l}\text { The business model } \\
\text { is very dependent } \\
\text { and/or based on } \\
\text { resource extraction }\end{array}$ & $\begin{array}{l}\text { The business model } \\
\text { is dependent on } \\
\text { resource extraction, } \\
\text { but is not based on it }\end{array}$ & $\begin{array}{l}\text { The business model is } \\
\text { dependent on some } \\
\text { resource extraction, but } \\
\text { facilitates the exchange } \\
\text { of existing resource }\end{array}$ & $\begin{array}{l}\text { The business model is } \\
\text { dependent on some resource } \\
\text { extraction, but facilitates the } \\
\text { exchange of existing } \\
\text { resource }\end{array}$ \\
\hline $\begin{array}{l}\text { Value is created by } \\
\text { a product or service } \\
\text { as opposed to } \\
\text { networks }\end{array}$ & $\begin{array}{l}\text { Users sharing ideas } \\
\text { and information with } \\
\text { one another as part } \\
\text { of an online network } \\
\text { that creates value }\end{array}$ & $\begin{array}{l}\text { Value is created by } \\
\text { users - consumers and } \\
\text { workers - sharing } \\
\text { access to underused } \\
\text { assets or human } \\
\text { resource as part of an } \\
\text { online network }\end{array}$ & $\begin{array}{l}\text { Value is created by } \\
\text { users - consumers and } \\
\text { workers - sharing access to } \\
\text { underused assets or human } \\
\text { resource as part of an } \\
\text { online, co-operative network }\end{array}$ \\
\hline \multirow{3}{*}{$\begin{array}{l}\text { Consumer and } \\
\text { workers exist in } \\
\text { silos and have } \\
\text { minimal power } \\
\text { over how } \\
\text { technology is used } \\
\text { to shape their lives } \\
\text { and work }\end{array}$} & $\begin{array}{l}\text { An intermediary } \\
\text { power connects } \\
\text { users and overseas } \\
\text { activity on a } \\
\text { platform }\end{array}$ & $\begin{array}{l}\text { An intermediary power } \\
\text { connects users and } \\
\text { overseas activity on a } \\
\text { platform }\end{array}$ & $\begin{array}{l}\text { No intermediary power is } \\
\text { needed to connect users and } \\
\text { oversee activity on a } \\
\text { platform, exchange is } \\
\text { genuinely peer-to-peer }\end{array}$ \\
\hline & $\begin{array}{l}\text { Users (consumers) } \\
\text { have some agency } \\
\text { over technology to } \\
\text { communicate with } \\
\text { one another and the } \\
\text { wider public }\end{array}$ & $\begin{array}{l}\text { Users empower } \\
\text { platforms and in turn } \\
\text { have the potential to be } \\
\text { better positioned in the } \\
\text { economy than previous } \\
\text { business models have } \\
\text { them allowed to }\end{array}$ & $\begin{array}{l}\text { Power is decentralized, } \\
\text { which is possibly facilitated } \\
\text { by blockchain technology }\end{array}$ \\
\hline & & $\begin{array}{l}\text { Users have some } \\
\text { agency over } \\
\text { technology to change } \\
\text { the way they live and } \\
\text { work }\end{array}$ & $\begin{array}{l}\text { Users (especially workers) } \\
\text { have agency over } \\
\text { technology to change how } \\
\text { they live and work in the } \\
\text { absence of an intermediary } \\
\text { power, particularly one } \\
\text { which presides over and } \\
\text { partakes in their earnings }\end{array}$ \\
\hline
\end{tabular}

Quelle: eigene Darstellung in Anlehnung an Balaram (2016)

ein möglichst dichtes Netz an einem vielseitigen Angebot zu haben, so richten sich die Marketingaktivitäten dahingehend aus, sowohl eine möglichst große Anzahl unterschiedlichster Gig-Worker und auch unterschiedlichster Auftraggeber auf der Plattform zu haben. Die Preisbildung und das Dienstleistungsangebot werden von der Plattform übernommen, deren Ziel es ist, die Dienstleistungsbuchungen möglichst zu steigern. Für die Ausübung der Gig-Worker-Tätigkeit spielen der Arbeitsort sowie die erforderlichen Qualifikationen eine entscheidende Rolle. Plattformen, die wie oben beschrieben operieren, 
sind darauf angewiesen, an den Orten, an denen ihre Dienstleistung angeboten wird, möglichst viele Gig-Worker im Einsatz zu haben. Solche Dienstleistungen erfordern meist keine hohen Qualifikationen. Dies würde eher einen Hinderungsgrund für den erfolgreichen Aufbau eines dichten Angebotes darstellen. Im Gegensatz zu diesem Geschäftsmodell gibt es Plattformen, die sich auf die Vermittlung von hoch qualifizierten Gig-Worker spezialisiert haben. Die angebotenen Dienstleistungen von diesen Talenten sind weniger ortsgebunden, sie können daher von verschiedenen Standorten aus für den Auftraggeber erledigt werden. Die Auftraggeber haben jedoch bei dieser Art von Gig einen Mehraufwand bei der Suche nach einem geeigneten Talent, da die Qualität der Arbeit stärker priorisiert wird als bei einer Massendienstleistung im Falle Ubers. Die Gewährleistung der geforderten Qualifikation und die Qualität der Arbeit ist ebenfalls für Gig-Worker zentral, die ihre Arbeitskraft auf einer solchen Plattform anbieten (Borell 2016). Diese Plattformen haben sich zum Ziel gemacht, hoch qualifizierte Gig-Worker mit anspruchsvollen Auftraggebern zu verbinden und müssen daher ihre Marketingaktivitäten auch dahingehend ausrichten.

\subsection{Lösungsansätze in der Theorie}

In diesem Kapitel werden die vorgestellten theoretischen Marketingkonzepte der 7 P's, der 4 C's und der 5 I's in Bezug auf Gig-Worker zusammengefasst. Onlinemarketing und die damit verbundene Erfolgsmessung werden ebenfalls in diesem Kapitel auf Lösungsansätze für Gig-Worker untersucht.

Sowohl theoretisch-generische Marketingkonzepte und deren Instrumente wie auch die Umwelt- und Marktanalysen sind zu einem großen Teil für das Marketing eines GigWorkers anwendbar. Die Herausforderung für den Gig-Worker dabei liegt darin, die einzelnen Ansatzpunkte exakt zu definieren, nach denen ein Marketingkonzept erstellt werden kann. Gig-Worker haben aufgrund der Plattformabhängigkeit nicht die Möglichkeit, alle I's, C's und P's gleichermaßen zu kontrollieren. Bei der Plattformwahl ist es entscheidend, dass Gig-Worker ihr Marketingkonzept bereits miteinbeziehen oder es gar nach den Plattformen ausrichten. Dieser Mehraufwand ist notwendig, da sonst kein einheitliches und aufeinander abgestimmtes Marketingkonzept erstellt werden kann. Einzelne P's lassen sich vom Inhalt her mit den entsprechenden C's und I's kombinieren (z. B. Price und Costs oder Promotion und Communication). Die Empfehlung der Theorie lautet daher, das Marketingkonzept basierend auf den jeweiligen Kombinationen zu erarbeiten.

Die Kombination der Marketingansatzpunkte gilt für das Offlinemarketing ebenso wie für das Onlinemarketing. Im Unterschied zu den Offlinemarketing-Maßnahmen gibt es beim Onlinemarketing spezifische Tools (z. B. SEO-Optimierungssoftware), Inhalte (z. B. Content Creation) und Prozesse (z. B. Conversion-Optimierung), die verstanden, erarbeitet und kontinuierlich bearbeitet werden müssen. Einen Vorteil, den das Onlinemarketing im Vergleich zu Offline-Maßnahmen und Instrumenten bietet, sind die Möglichkeiten einer detaillierten Erfolgsmessung. Online-Maßnahmen können direkt auf ihre 
Wirkung und damit auf den Nutzen abgestimmt werden. Demgegenüber steht das erhöhte Risiko des Kontrollverlustes über veröffentlichte Inhalte im Internet und der damit verbundene Mehraufwand der Kontrolle und Bewirtschaftung der Onlinekanäle.

\subsection{Methoden}

Damit die Zielsetzung der Arbeit bestmöglich erreicht werden konnte, musste ein Forschungsdesign ausgewählt werden, welches erlaubt, das Phänomen der Gig-Arbeit aus unterschiedlichen Perspektiven empirisch zu untersuchen. Es sollte sowohl die Sicht der Gig-Worker, diejenige der Plattformen wie auch die der Auftraggeber respektive der Kunden dieser Plattformen berücksichtig werden. In einer Kombination zweier induktiver Ansätze, nämlich von Fallstudien gemäß dem Case Study Research Process (Reddy 2015; Yin 2014) und Expertenworkshops, wurden der Forschungsproblematik Rechnung getragen. In einem 1. Schritt wurden Fallstudien von Gig-Workern und Auftraggebern erarbeitet. Anschließend wurden aufgrund der Fallstudienergebnisse Expertenworkshops durchgeführt. Im Folgenden werden diese beiden Vorgehensweise näher erläutert.

\subsubsection{Fallstudien}

Die Selektion der Fallstudien sollte einem möglichst breiten Spektrum verschiedenster Anbieter, sowohl von Dienstleistungen wie auch Waren, gerecht werden. Zudem wurde darauf geachtet, individuelle Anbieter wie auch Unternehmen mit mehreren Mitarbeitern in der Stichprobenselektion zu berücksichtigen. Mit dieser breiten Streuung verschiedenster Dienstleistungs- und Warenanbieter konnten deren unterschiedliche Bedürfnisse und Ressourcen berücksichtigt werden. Schließlich wurden Auftraggeber ausgewählt, die ebenfalls unterschiedliche Leistungen suchten. Dabei sollten sie jedoch passende Leistungen suchen, sodass durchaus ein reales Auftragsverhältnis zu den Anbietern hätte entstehen können.

Der semistrukturierte Leitfaden für die Gesprächsführung entstand anhand der SPSSMethode Sammeln, Prüfen, Sortieren und Subsumieren (Helfferich 2014, S. 559-575). Die in den vorhergehenden Kapiteln beschriebene Theorie dient dabei als Orientierungshilfe. Zur Konzipierung der Fragen wurden die 10 Gebote von Porst (2014) berücksichtigt. Dabei musste insbesondere darauf geachtet werden, den Gesprächspartner nicht zu verunsichern und ihn in seinen Aussagen auch nicht zu sehr zu steuern. Der Gesprächsleitfaden wurde nach seiner selbstständigen Erstellung durch einen Marketingexperten der Firma Innoarchitects AG in Bern getestet. Dabei wurde die 2. Forschungsfrage zur Identifikation von Schlüsselfaktoren ausführlich in Gesprächsfragen umformuliert. Mit jeweils 2 Fragen wurden die 7 P's, 4 C's und 5 I's besprochen. Die 3. Forschungsfrage zur Nachfragensteigerung wurde einerseits mit Fragen zum Onlinemarketing und andererseits mit Fragen zum generellen Inhalt eines Marketingkonzeptes untersucht. 
Tab. 14.2 Codierung Leitfadeninterviews

\begin{tabular}{l|l|l|l|l}
\hline Warm-up & RQ1 & RQ2 & RQ3 & Abschluss \\
\hline Warm-up: & RQ1: Auswahl Gig- & RQ2: Communication & RQ3: Marketing- & Abschluss \\
Persönliche & Worker & RQ2: Convenience & budget & Ergänzungen \\
Angaben & RQ1: Erfolgsfaktoren & RQ2: Cost to Customer & RQ3: Marketing- & \\
Warm-up: & RQ1: Marketing & RQ2: Identification & inhalt & \\
Selbstbe- & Stellenwert & RQ2: Individualization & RQ3: & \\
zeichnung & RQ1: & RQ2: Integration & Marketingmaßnah- & \\
Warm-up: & Marketingmaßnah- & RQ2: Integrity & men & \\
Gefallen an & men & RQ2: People & der Plattformen & \\
Gig- & RQ1: Over-/ & RQ2: Physical & RQ3: SEO & \\
Worker & Underperformer & Evidence & RQ3: Social Media & \\
& RQ1: Suche nach & RQ2: Place & Marketing & \\
& Gig- & RQ2: Price & RQ3: & \\
& Worker & RQ2: Process & Wirkungspotenzial & \\
& RQ1: Vergleich mit & RQ2: Product & Marketing & \\
& anderen Gig-Worker & RQ2: Promotion & & \\
& RQ1: Vorhandensein & RQ2: Überprüfung & & \\
& Marketingkonzept & Marketingmaßnahmen & & \\
\hline
\end{tabular}

Quelle: eigene Darstellung

Für die Datenauswertung wurde die Software Atlas TI verwendet. Die Wort für Wort transkribierten Fallstudiengespräche wurden anhand der einzelnen Forschungsfragen und Untersuchungszielen jeweils mit Codes versehen. In Tab. 14.2 kann man die dabei erstellte Codierungsliste sehen.

\subsubsection{Expertenworkshops}

Die Expertenworkshops dienten dazu, zusätzlich zu den Auftraggebern und -nehmern die Expertise der Plattformenbetreiber auf das Thema miteinzubeziehen. Dazu wurde ein rund 3-stündiger Expertenworkshop durchgeführt. Die Leitthemen des Workshops lauteten: (a) die Notwendigkeit eines Marketingkonzeptes für die Gig-Worker zu ermitteln, (b) Richtlinien für die Erstellung eines Marketingkonzeptes für die Gig-Worker festzulegen und (c) die Rolle der Plattformen zu erfassen und gegebenenfalls zu optimieren.

Die Experten hatten 15 Minuten Zeit, die Notwendigkeit eines Marketingkonzeptes zu definieren. Dabei mussten sie eine Vierfeldermatrix erstellen und deren Achsen und Felder bestimmen. Anschließend wurden die Ergebnisse diskutiert und in Einstimmigkeit zu allen Teilnehmern festgelegt. Zur Erstellung der Richtlinien des Marketingkonzeptes wurden individuelle Brainstorming-Runden von je 5 Minuten vereinbart. Anschließend wurden wiederum im Plenum die Kernaussagen zu den theoretischen Marketingkonzepten und deren Bezug auf Gig-Worker zusammengetragen. Zu guter Letzt wurden, wiederum im Plenum, alle Kernaussagen zu jeweils einer Hauptrichtlinie pro MarketingkonzeptBestandteil reduziert. 


\subsection{Lösungsansätze für die Praxis}

Gig-Worker müssen sich entscheiden, ob sie als Person oder Unternehmen auftreten möchten. Je nach gewähltem Auftritt werden andere Zielgruppen angesprochen. Private Gig-Worker führen Aufträge für Privatpersonen und Unternehmen aus, während Gig-Worker die als Marke oder Unternehmen auftreten hauptsächlich nur Aufträge für Unternehmen ausführen. Bei allen Auftritten muss jedoch ein übersichtliches, vollständiges und gut bewertetes Profil auf der Plattform vorhanden sein. Die Offerte eines Gig-Workers muss professionell gestaltet sein. Der offerierte Preis soll einerseits der gebotenen Leistung entsprechen und sich andererseits nicht auffallend von den Preisen anderer Gig-Worker unterscheiden. Generell ist der Preis eines Gig-Workers niedriger als derjenige für dieselbe Leistung, die von einem Unternehmen erbracht wird. Der Kunde bestimmt die zeitlichen Rahmenbedingungen des Auftrages. Der Arbeitsort ist dabei abhängig von der Art der Tätigkeit, die der Auftrag erfordert. Eine persönliche Kommunikation vom Gig-Worker mit den Auftraggebern bringt Vorteile in der Effizienz der Arbeitserledigung und hilft bei der Kundenbindung.

Standardisierte Prozesse helfen Gig-Workern, zeitlichen und finanziellen Aufwand zu vermindern. Auftraggeber dürfen aber dadurch nicht das Gefühl kriegen, an individueller Betreuung zu verlieren. Sowohl Gig-Worker wie auch Auftraggeber haben Vertrauen in die Dienstleistungen der Plattformen. Diese Sicherheit der Plattformen trägt dazu bei, dass sowohl die Gig-Worker und dessen Auftraggeber von einem gegenseitigen Grundvertrauen profitieren können.

Das größte Wirkungspotenzial wird Marketingmaßnahmen im Internet zugesprochen. Vor allem Gig-Worker, die Produkte und keine Dienstleistungen anbieten, müssen nebst ihren Profilen auch eine im Internet basierte Verkaufsmöglichkeit anbieten können. Der persönliche Kontakt mit Auftraggebern wird vor allem bei angebotenen Dienstleistungen erwähnt. Dieser hilft, das benötigte Vertrauen zwischen den Parteien herzustellen und auch zu stärken. Plakate und ähnliche physische Marketingmaßnahmen wurden von den Studienteilnehmern als nichtrelevant bezeichnet. Marketingmaßnahmen im Social-MediaBereich würden gerne betrieben werden. Ein Hinderungsgrund ist jedoch die Schwierigkeit, mit geeigneten Inhalten aus der Masse herauszustechen und sich gleichzeitig so zu präsentieren, dass auch mit möglichen direkten, negativen Reaktionen umgegangen werden muss. Ein weiteres Kriterium für Social-Media-Marketing ist die Zielgruppe, die durch den Gig-Worker angesprochen werden möchte. Soziale Medien erlauben das gezielte Verbreiten von Marketingmaßnahmen, sind jedoch in ihrer Erstellung aufwendig. Damit Social-Media-Marketing erfolgreich ist, muss eine möglichst breite Masse mit dessen Inhalten angesprochen werden können. Abhängig von der Tätigkeit eines Gig-Workers existiert aber nicht zwingend die erforderliche Menge an potenziellen Auftraggebern, die das Betreiben von Social-Media-Marketing rechtfertigen würde.

Auftraggeber haben einheitlich berichtet, dass sie durch Marketingmaßnahmen der Plattform überhaupt erst dazu gekommen sind, Aufträge an Gig-Worker zu vergeben. Je aktiver eine Plattform Marketingmaßnahmen betreibt, umso besser wirkt sich dies auf die Buchungsanzahl ihrer Talente aus. Bei der Plattformwahl müssen sich Gig-Worker daran orientieren, welche Plattform den höchsten Mehrwert im Marketing bietet, um dadurch mehr 
Auftraggeber auf ihre Plattform zu leiten. Bereits registrierte Auftraggeber wollen aber nach der Registrierung nicht mit Marketingmaßnahmen übersättigt werden. Das richtige Maß an Marketingmaßnahmen zu finden, liegt in der Verantwortung der jeweiligen Plattform.

Der von Auftraggebern gewünschte Inhalt der Marketingmaßnahmen bezieht sich auf die Informationen, die der Gig-Worker auf seinem Profil bereitstellt. Selten werden Marketingmaßnahmen neben dem eigentlichen Plattformprofil angeschaut oder gar in den Auswahlentscheidungsprozess miteinbezogen. Ein übersichtliches, vollständiges und aktuelles Profil ist dabei entscheidend. Je nach Tätigkeit des Gig-Workers kann ein Logo oder aber ein persönliches Foto der Person vorteilhafter sein. Wie in der Auswertung der 1. Forschungsfrage ist auch das Vorhandensein und die Präsentation der Referenzen als geforderter Inhalt von Marketingmaßnahmen als zentral erachtet worden.

Die eigene Arbeit wird von Gig-Workern als Hauptinhalt verwendet, um zukünftige Auftraggeber von sich zu überzeugen. Dieser muss aber auf dem Plattformprofil wie auch auf anderen Kanälen einheitlich präsentiert werden. Auftraggeber entscheiden bei der Auswahl des Gig-Workers auch aufgrund ihres ersten Eindrucks. Wird dieser als positiv empfunden, werden im nächsten Schritt die Zeit in der der Gig-Worker antwortet, sein Erscheinungsbild sowie der genannte Preis der Offerte bzw. die Art und Weise der Kommunikation als weitere wichtige Kriterien erwähnt.

Das Konzept des SEO (Search Engine Optimization) wird von Gig-Workern, die eine eigene Webseite unterhalten, als wichtig angesehen. Ein Hinderungsgrund dabei ist jedoch das Wissen und die Fähigkeit zur Umsetzung des Konzeptes. Die Integration von SEO muss bereits bei der Erstellung der Webseite berücksichtigt werden. Der Aufwand einer nachträglichen Einführung ist bedeutend größer. SEO-Maßnahmen sind für die Steuerung des Suchprozesses von Auftraggebern außerhalb der Gig-Plattformen eine entscheidende Maßnahme. Ein definiertes Marketingbudget oder gezielte Rücklagen für Marketingmaßnahmen werden von Gig-Workern wenig bis gar nicht vorgenommen. Gewinne aus der Tätigkeit des Gig-Workers werden unter anderen für den Zukauf und Amortisation etwaiger Ausrüstungen und zum generellen Ausbau der unternehmerischen Tätigkeiten verwendet.

Als erfolgreiche Marketingmaßnahmen bewerteten die Gig-Worker, wenn die Plattformen hohe Bewertungen haben, die Präsenz des eigenen Logos auf Produkten und die Förderung von Mund-zu-Mund-Propaganda. Vor allem bei Gig-Tätigkeiten, die einen persönlichen Kontakt zwischen den Parteien verlangen, sind die persönliche Erscheinung und der erste Eindruck wichtige Instrumente. Ein professionelles Auftreten während der gesamten Prozessdauer und über alle Kanäle hinweg ist für eine gute Bewertung seitens der Auftraggeber essenziell.

\subsubsection{Marketing-Guidelines für Gig-Worker}

In den Bereichen Product, Clients und Identification sollten sich die Gig-Worker bewusst sein, dass sie neben ihrer Tätigkeit auch ihre Person vermarkten müssen. Die Kernaussage hier könnte also lauten: „Nicht nur mein Angebot, sondern auch meine Person können das Produkt sein." Es ist dabei jedoch ein Unterschied, ob sie als Privatperson oder als Unter- 
nehmen auf der Plattform auftreten. Sie sollten demnach nebst der Qualität ihrer Arbeit auch auf die Qualität ihrer Präsentation von sich selbst achten. Ihr Ziel ist es dabei, dass sich die Auftraggeber mit dem angebotenen Gesamtpaket aus Tätigkeit und Person identifizieren können.

Bei den Kosten respektive dem Preis, also Price und Cost, wurde die Kernaussage „Die Kompetenzen des Gig-Workers und die Strategie bestimmen den Preis.“ definiert. Inwiefern die Kompetenzen des Gig-Workers den Preis bestimmen, kann anhand eines Praxisbeispiels verdeutlicht werden: Eine Studentin im Fach Grafikdesign, die noch über kein abgeschlossenes Studium verfügt, jedoch genügend Zeit hat, ein paar Aufträge entgegenzunehmen, um damit auch Berufs- und Praxiserfahrung zu sammeln, verlangt einen wettbewerbsfähigeren Preis als eine diplomierte Grafikerin mit viel Berufserfahrung. Sie wird sich eher als individuelle Arbeitskraft auf der Plattform präsentieren und nicht als Firma auftreten. Ihre Kunden werden eher Privatpersonen und nicht Unternehmen sein. Für sie wird das Honorar einer professionellen Agentur zu teuer sein. Im Gegensatz dazu sind professionelle Grafikagenturen in einem höheren Preissegment angesiedelt und werden einen professionelleren Auftritt auf der Plattform haben und ihren Auftritt hauptsächlich zur Steigerung der Zahl der Aufträge nutzen. Der Preis und die zusätzlichen Kosten, die für den Kunden entstehen, entscheiden darüber, welche Kunden vom Gig-Worker gewonnen werden können. Basierend auf der Strategie und der aktuellen Situation kann der Gig-Worker den Preis so setzen, dass er die langfristige Erreichung seiner Ziele unterstützt. Aufbauend auf der 1. Richtlinie sind damit der Preis und das Produkt gleichermaBen gewichtet und auf die geeigneten Kunden ausgerichtet.

Zum Thema Place und Convenience wurde die Kernaussage „Der Kunde ist da wo er mich braucht.“ definiert. Die angebotenen Tätigkeiten auf Gig-Plattformen unterscheiden sich auch hinsichtlich ihrer Ortsabhängigkeit. Der Rasen eines Auftraggebers muss natürlich bei ihm oder ihr zu Hause gemäht werden, der Ort ist vorgegeben. Eine Übersetzung eines Textes in eine andere Sprache kann über das Internet und Plattformen von überall aus erledigt werden, der Ort ist also nicht vorgegeben.

Für Gig-Worker ist es demnach entscheidend, dass sie ihr Angebot so platzieren, dass sie genau da sind, wo der Kunde sie braucht. Dementsprechend soll der Auftraggeber sie schnell bei der Suche nach geeigneten Talenten finden.

In den Bereichen Promotion, Communication, Indiviualization und Interaction gilt das Kernprinzip: „Je komplexer, desto individueller.“ Die Komplexität der angebotenen Leistung und der Aufträge spielt eine entscheidende Rolle darin, wie auf Kunden zugegangen, kommuniziert und interagiert werden sollte. Das Gesamtziel ist, dass sich alle Kunden individuell angesprochen und behandelt fühlen und eine persönliche Lösung für ihre Anliegen geboten werden kann. Diese Richtlinie besagt, dass mit zunehmender Komplexität auch der Aufwand des Gig-Workers steigt. Der Gig-Worker ist nach diesen 4 Richtlinien nicht nur da wo der Kunde ihn braucht, sondern interagiert auch kundengerecht.

In der Kategorie Process wurde die Kernaussage ,Der Kunde bestimmt den Prozess: Einfach, transparent und nachvollziehbar.“ definiert. Wie die vorangegangenen Richtlinien ist auch diese am Kunden orientiert. Die mögliche Flexibilität von Gig-Workern hat neben den individuellen Vorteilen auch die Anforderungen der Kunden zu berücksichti- 
gen. Der Prozess von der Suche nach dem geeigneten Talent bis zum Abschluss des Auftrages, aber auch die Nachbearbeitung wird vom Kunden bestimmt. Das Befolgen der vorangegangenen Richtlinien stellt für Gig-Worker die Grundvoraussetzung dar, für den Kunden den Gesamtprozess so angenehm wie möglich zu gestalten.

In Bezug auf People, Integrity und Integration gilt das Prinzip „Die Menschen kennen und teilen meine Werte und umgekehrt.“ Die Arbeit in der Gig Economy ermöglicht es Talenten und Auftraggebern aus allen Bereichen, Ländern, Kulturen und Zeitzonen, noch intensiver und direkter als bisher zusammenzuarbeiten. Die unterschiedlichen Wertvorstellungen zusammenzuführen bringt einige Herausforderungen mit sich. Gig-Worker müssen in der Lage sein, ihre Wertvorstellungen klar zu definieren. Sie sollten zudem auch bereit sein, diese zu verteidigen, ohne dabei die Wertvorstellungen der Auftragspartei zu verletzen. Ziel ist es, dass sowohl beide Parteien als auch alle weiteren involvierten Menschen und Organisationen zu dem gewünschten Resultat kommen, ohne dabei die Wertvorstellungen zu kompromittieren, die Integrität zu behalten und durch Integration noch näher zusammenwachsen.

Bei dem Themenbereich Physical Evidence gilt die Kernaussage „Ich bin mir bewusst, dass ich die Marke bin. "Übergreifend auf alle Richtlinien sollte sich ein Gig-Worker bewusst sein, dass alle Tätigkeiten (Arbeit, Werbung, Auftreten etc.), die von der Umwelt wahrgenommen werden können, eine Wirkung erzeugen. Diese Wirkung muss so gut wie möglich durch den Gig-Worker gesteuert werden. Dabei ist es hilfreich, wenn die gesamte Präsentation der Person, des Produktes, des öffentlichen Auftritts, der Onlinekanäle und der Marketinginstrumente einheitlich gestaltet wird. Dadurch lässt sich ein gesteuertes Gesamtbild erschaffen, das für die unterschiedlichen Interessensgruppen gleichermaßen passend ist. Die einheitliche Wahrnehmung hilft zudem dabei, den Wiedererkennungswert und damit auch die Marke zu steigern.

Schlussfolgernd lässt sich sagen, dass die 7 Guidelines den Gig-Worker eine gute Grundlage bieten, nach welcher sie ihr Marketingkonzept und die entsprechenden Marketinginstrumente und Inhalte gestalten können. Die generische Formulierung verlangt allerdings, dass jeder Gig-Worker individuell diese Überlegungen auf sich und seine Tätigkeit beziehen sollte, um daraus das Marketingkonzept erstellen zu können. Entscheidend über den gewünschten Erfolg sind aber das Zusammenspiel und die Abhängigkeit der einzelnen Richtlinien untereinander. Diese dürfen in der Ausführung nicht in Widerspruch zueinanderstehen, sondern sollten sich gegenseitig bestätigen und stärken.

\subsubsection{Folgerungen aus der praktischen Untersuchung}

Die Untersuchung hat gezeigt, dass die im theoretischen Teil dieser Arbeit vorgestellten Marketingkonzepte, Instrumente und Maßnahmen auch von Gig-Workern anwendbar sind, um ihr persönliches Marketingkonzept zu erstellen. Das finanzielle und professionelle Ziel, welches durch die Gig-Tätigkeit verfolgt wird, entscheidet dabei über den Aufwand, den ein Gig-Worker bereit ist, in Marketing zu investieren. Die Unterscheidung zwischen Over- und Underperformer kann nicht nur in Bezug auf das jeweilige Marketingkonzept vorgenommen werden, sondern hängt mit weiteren Faktoren zusammen. 
Gig-Worker die auch außerhalb der Plattform(en) sich durch eine eigene Webseite, Social Media, Flyer, Produkte oder öffentliche Auftritte präsentieren, betreiben damit einen höheren Aufwand im Marketing als Gig-Worker, die nichts von alledem unternehmen. Sie sind es auch, die eine finanzielle Abhängigkeit von ihrer Tätigkeit haben und GigWork als Teil ihres unternehmerischen Wirkens betreiben können.

Die eindeutige Identifikation von Schlüsselfaktoren im Marketingkonzept von Schweizer Gig-Workern ist aufgrund der vielseitigen Tätigkeiten, Ansprüche und Ziele der jeweiligen Gig-Worker schwierig. Allgemein gültige Schlüsselfaktoren konnten jedoch erhoben werden. Die Wahl der richtigen Plattformen aufgrund ihrer angebotenen Dienstleistungen, die Anzahl verfügbarer Aufträge auf der Plattform und die von der Plattform betriebenen Marketingmaßnahmen sind entscheidend. Ein vollständiges, übersichtliches, mit Referenzen und Anschauungsmaterial versehenes Profil, welches mit einem professionellen Foto/ Logo als Profilbild geführt wird, muss vom Gig-Worker gepflegt werden. Eine zuverlässige, rasche und unkomplizierte Erledigung der Aufträge führt zu einer guten Bewertung auf der Plattform und hebt Gig-Worker von anderen ab. Eine persönliche Kommunikation mit Auftraggebern stärkt das Vertrauen zwischen den Parteien. Gig-Worker müssen in der Lage sein, die vom Kunden gewünschten Kommunikationskanäle verwenden zu können und eine schnelle Reaktionszeit aufweisen. Ein professioneller Auftritt vor Ort, persönlicher Kontakt und individuelle Betreuung der Auftraggeber helfen dabei, langfristige Geschäftsbeziehungen aufzubauen und fördern die Weiterempfehlungsrate von Gig-Worker. Der Preis muss so gestaltet werden, dass dieser für die gewünschte zu erreichende Zielgruppe an potenziellen Auftraggeber mit deren geforderten Leistung übereinstimmt.

Marketingmaßnahmen mit dem höchsten Wirkungspotenzial sind neben dem Profil und dessen Bewertung die Referenzen eines Gig-Workers, Mund-zu-Mund-Propaganda unter den Auftraggebern sowie die Qualität der geleisteten Arbeit.

\subsection{Empfehlungen}

Ziel dieser Arbeit ist es, eine generische Vorlage zur Erstellung eines Marketingkonzeptes durch Gig-Worker in der Schweiz zu liefern. Basierend auf den vorgestellten theoretischen Konzepten und in Kombination mit den Ergebnissen der Fallstudien und des Expertenworkshops wurde eine solche Vorlage erstellt. Diese Vorlage wird nicht für alle Gig-Worker den gleichen Nutzen stiften können. Je nach Tätigkeit, Zielsetzungen und aktuellem Marketingkonzept muss ein Gig-Worker sich in dieser Vorlage einordnen und für sich abwägen, welche Inhalte für das persönliche Marketingkonzept relevant sind und wie diese umgesetzt werden sollen. Gig-Worker sollen diese Vorlage als Ausgangssituation verwenden und mit weiteren Ergebnissen aus den Teilbereichen dieser Arbeit sowie eigenen Ansätzen ergänzen, um ein eigenes Marketingkonzept zu erstellen.

Abb. 14.1 zeigt in der obersten Zeile die im Expertenworkshop erarbeiteten Guidelines für die jeweiligen Teilbereiche im Marketingkonzept. Diese Richtlinien beschreiben die Grundeinstellung, die Gig-Worker für diesen Teilbereich einnehmen sollen. Die Marke- 


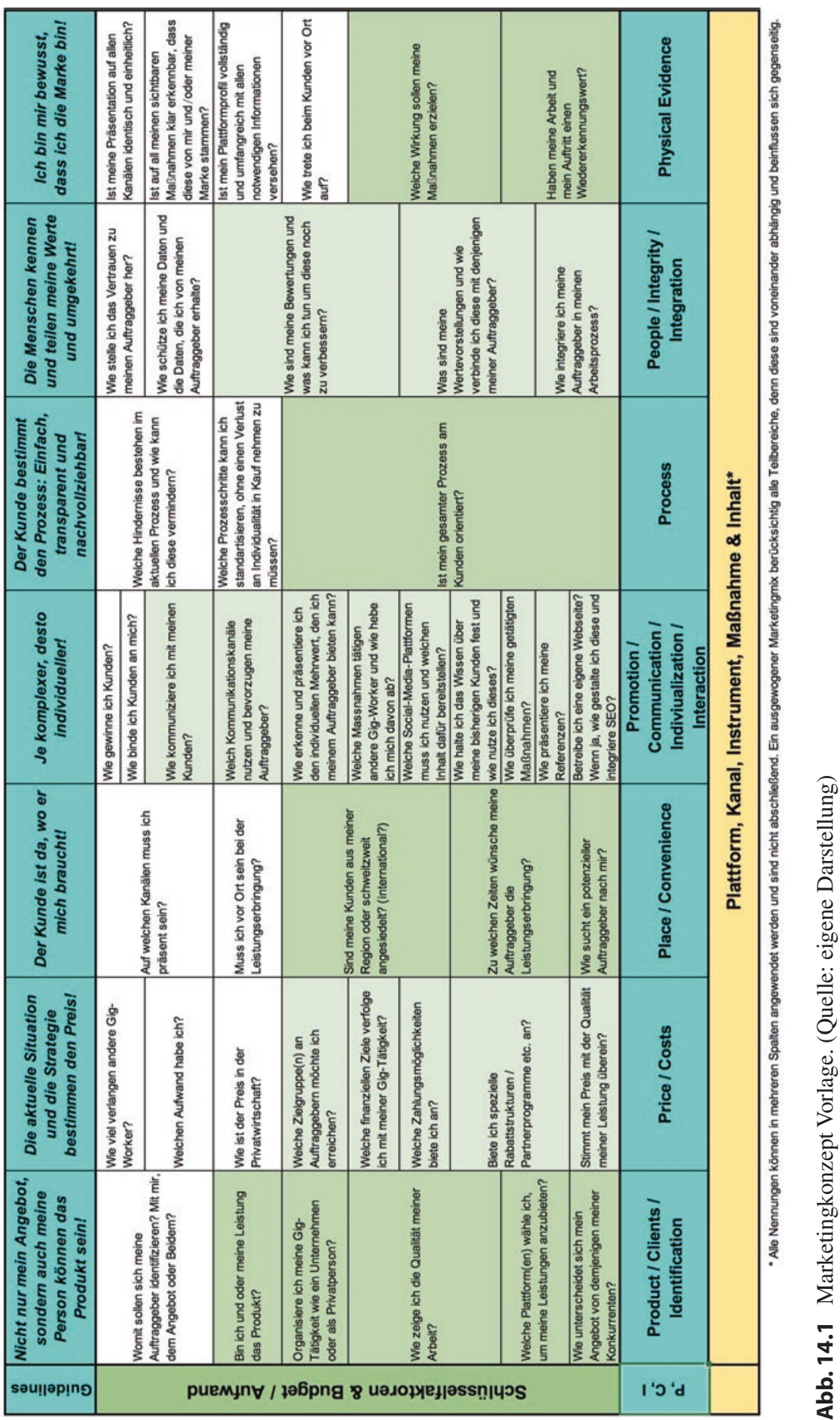


tingteilbereiche sind in derselben Farbe wie deren Richtlinien in der Tabelle markiert und füllen die zweitunterste Zeile aus. Im Hauptteil folgen die in dieser Arbeit identifizierten Schlüsselfaktoren. Formuliert sind diese als Fragen zu den jeweiligen Marketingkonzeptbereichen, die Gig-Worker bei der eigenen Konzepterarbeitung beantworten müssen. Diese Fragen sind nicht abschließend und in der Tabelle jeweils nur einem Bereich zugeordnet. Sie sind jedoch als bereichsübergreifend anwendbar zu verstehen. Ein erfolgreicher Marketingmix basiert darauf, dass Maßnahmen, Instrumente und Inhalte aller Teilbereiche aufeinander abgestimmt und kongruent betrieben werden. Diese Zusammenhänge werden durch die unterste Zeile der Tabelle in Orange dargestellt. Die grüne Markierung der Felder im Hauptteil der Tabelle soll in ihren unterschiedlichen Höhen visualisieren, dass Gig-Worker ihren Aufwand, den sie für den jeweiligen Teilbereich im Marketing aufwenden. einschätzen müssen. Je nach aktuellem Stand muss ein Gig-Worker für einzelne Bereiche noch mehr Aufwand betreiben, um einen ausgewogenen Marketingmix zu erhalten.

\section{Literatur}

Balaram, B. (2016). A. Medium corporation. RSA Reports, Royal Society for the encouragement of Arts, Manufactures and Commerce (13. Januar 2016). https://medium.com/rsa-reports/fairshare-reclaiming-power-in-the-sharing-economy-499b46bd4b00. Zugegriffen im März 2018

Borell, N. (2016). Das Industrie 4.0 Arbeitsbuch: Sind Digitalisierung, Industrie 4.0 und Disruption unterschiedliche Dinge?! Hamburg: tredition $\mathrm{GmbH}$.

Bürgi, Nägeli Rechtsanwälte. (01. Januar 2008). Freier Mitarbeiter. LawMedia AG. Freelancer/ Freier Mitarbeiter/Freischaffender Mitarbeiter. https://www.freier-mitarbeiter.ch/begriff. Zugegriffen im März 2018

Burri, T., Figini, R., \& Rosa, S. (2018). Arbeitsbiographien in der Gig-Economy. Berner Fachhochschule für Wirtschaft und Verwaltung. Bern: Nicht publiziert.

Deloitte. (2016). Deloitte Development LLC (25.08.2016). Deloitte Development LLC. https:// www2.deloitte.com/us/en/pages/about-deloitte/articles/press-releases/deloitte-surveyworkplace-culture-important-to-independent-contractors.html?nc=1. Zugegriffen im März 2018

Helfferich, C. (2014). Leitfaden- und Experteninterviews, Handbuch Methoden der empirischen Sozialforschung. Wiesbaden: Springer Fachmedien.

Jaekel, M. (2017). Die Macht der digitalen Plattformen: Wegweiser im Zeitalter einer expandierenden Digitalsphäre und künstlicher Intelligenz. Wiesbaden: Springer.

Mattmann, M., Walther, U., Frank, J., \& Marti, M. (2017). Die Entwicklung atypisch-prekärer Arbeitsverhältnisse in der Schweiz. Bern: Staatssekretariat für Wirtschaft SECO.

McGovern, M. (2017). Thriving in the Gig economy: How to capitalize and compete in the new world of work. Wayne: The Career Press Inc.

Merath, S. (2008). Der Weg zum erfolgreichen Unternehmer. Offenbach: GABAL.

Oakley, D. (2016). Business Model Guru (27.01.2016). Business Model Guru. http://www.businessmodel.guru/the-uber-business-model-canvas/. Zugegriffen im März 2018

Osterwalder, A., \& Pigneur, Y. (2011). Business model generation. Frankfurt: Campus.

Porst, R. (2014). Fragebogen, Ein Arbeitsbuch. Wiesbaden, Deutschland. Berlin: Springer.

Reddy, K. (2015). Sciencedirect (01.12.2015). https://www.sciencedirect.com/science/article/pii/ S2314721015000031. Zugegriffen im März 2018 
Risak, M., \& Lutz, D. (2017). Arbeit in der Gig-Economy, Rechtsfragen neuer Arbeitsformen in Crowd und Cloud. Wien: ÖGB.

Schöchli, H. (30. August 2016). Neue Zürcher Zeitung AG. https://www.nzz.ch/wirtschaft/unternehmen/plattform-wirtschaft-uber-bangt-um-sein-erfolgsmodell-ld.113662.Zugegriffen im März 2018

Shahdad, S., \& Seidenstein, T. (2017). Fannie Mae (05.12.2017). http://www.fanniemae.com/portal/research-insights/perspectives/gig-economy-homeownership-120517.html. Zugegriffen im März 2018

Stähler, P. (2002). Geschäftsmodelle in der digitalen Ökonomie: Merkmale, Strategien und Auswirkungen. Zürich: EUL.

Upwork Global, I. (2018). Upwork Global Inc (17.03.2018). www.upwork.com. Zugegriffen im März 2018

UpWork, I. (2015). Elance and oDesk databases. http://elance-odesk.com/online-work-reportglobal. Zugegriffen im März 2018

Yin, K. R. (2014). Case study research, design and methods (Bd. Bd. 5). Los Angeles: SAGE Publications, Inc.

Tobias Burri, Masterstudium in Betriebswissenschaft an der Berner Fachhochschule mit Vertiefung in Corporate \& Business Development. Tätigkeit als Project Manager, Innovation Co-Creator bei der INNOArchitects AG und Geschäftsstellenleiter „ActionJam“, dem größten Corporate-Netzwerk für Innovationsmanagement der Schweiz.

Dr. E. J. Rumo ist assoziierter Professor für Marketing und Sales Management an der Hochschule für Wirtschaft in Freiburg (HES-SO) und Dozent am konsekutiven Masterstudiengang der Berner Fachhochschule mit Studien (MBA) an der University of Edinburgh (UK) und (PhD) der Université de Lorraine (France). Seine Forschungsaktivitäten konzentrieren sich auf Sales Management und International Entrepreneurship. Seit über 10 Jahren begleitet er als Berater KMU's und Großbetriebe in den Bereichen Verkauf, Vertrieb und International Business Development.

Open Access Dieses Kapitel wird unter der Creative Commons Namensnennung 4.0 International Lizenz (http://creativecommons.org/licenses/by/4.0/deed.de) veröffentlicht, welche die Nutzung, Vervielfältigung, Bearbeitung, Verbreitung und Wiedergabe in jeglichem Medium und Format erlaubt, sofern Sie den/die ursprünglichen Autor(en) und die Quelle ordnungsgemäß nennen, einen Link zur Creative Commons Lizenz beifügen und angeben, ob Änderungen vorgenommen wurden.

Die in diesem Kapitel enthaltenen Bilder und sonstiges Drittmaterial unterliegen ebenfalls der genannten Creative Commons Lizenz, sofern sich aus der Abbildungslegende nichts anderes ergibt. Sofern das betreffende Material nicht unter der genannten Creative Commons Lizenz steht und die betreffende Handlung nicht nach gesetzlichen Vorschriften erlaubt ist, ist für die oben aufgeführten Weiterverwendungen des Materials die Einwilligung des jeweiligen Rechteinhabers einzuholen.

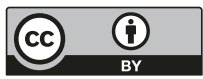

